\title{
SUBJECTIVE PROBABILITIES FOR STATE DEPENDENT CONTINUOUS UTILITY*
}

\author{
Peter WAKKER \\ Deparment of Mathematics. University of Vijmegen, 6525 ED Nimegen. The Verhertands
}

Communicated by Peter Fishburn

Received 15 January 1986

Revised 29 September 1986

\begin{abstract}
For the expected utility model with state dependent utilities, Karni, Schmeidler and Vind (1983) have shown how to recover uniquely the involved subjective probabilities if the preferences, contingent on a hypothetical probability distribution over the state space, are known. This they do for consequence spaces, consisting of lotteries on sets of prizes. We adapt their work to consequence spaces that are connected topological spaces, without using lotteries on them. E.g. our consequences may be monej, or commodity bundles.
\end{abstract}

Key words: Subjective probability; state dependent expected utility; additive representation.

\section{Introduction}

One of the restrictions for the applicability of the usual expected utility model is that in many cases the consequences are thus state-specific, that the usual state independence is inconceivable. Already Ramsey (1931), the first derivation of subjective expected utility, indicated that events may very well not be ethically neutral, which comes down to the same thing as state dependence. Some references, examples, and applications of state dependent expected utility are Eisner and Strotz. (1961), Section IV in Yaari (1969), Arrow (1974), Cook and Graham (1977). For further references, and many results, see Karni (1985).

If state dependent utilities are permitted, then, without further information, the factors probability and utility can no longer be separated in a unique way. Any change in probabilities, not affecting the positivity of them, can be handled by a multiplication of utilities with appropriate factors. In Karni, Schmeidler and Vind (1983), hereafter abbreviated KSV, it is indicated how, with the further information of the preferences contingent on a hypothetical probability distribution, the factors probability and utility can be separated in a unique way, under a consistency

\footnotetext{
* This research has been done at the Department of Statistics at the University of Tel Aviv. I gratefully acknowledge financial support from the Netherlands Organization for the Advancement of Pure Rescarch (Z.W.O.). 
axiom: Thus KSV obtain a derivation of expected utility wh state dependent utility. This result has been derived for the case where consequences are lotteries on a finite set. It can without any problem be extended to mixture spaces (for a definition see Herstein and Milnor, 1953) as consequence spaces, as long as utility is linear. So their approach not only handles the case where consequences are lotteries, but also the case $w$ here consequences are commodity bundles (or money) with linear utility.

In this paper we adapt the work of KSV to the case where the consequence spaces are connected topological spaces, and utility only has to be continuous. Thus we handle for instance the case where consequences are commodity bundles (or money), and utility is not (necessarily) linear. The price for this generalization is that "We must replace 'weak consistency', the appealing characterizing condition of KSV, by 'cardinal consistency', a more complicated condition. The latter condition is still necessary (and sufficient) though, and in the KSV set-up quickly can be derived from linearity and ordinal consistency (see Proposition 4.2).

\section{Definitions, notations, and preliminary results}

Let $S=\left\{s_{1}, \ldots, s_{n}\right\}$ be a finite state space. Let for every $s$, be given a consequence space $\psi_{i} . L=X_{j=1}^{n} \gamma_{j}$ is the set of acts. Act $x=\left(x_{1}, \ldots, x_{n}\right) \in L$ assigns $x_{j}$ to $s$, $i-1, \ldots, n$. The (actual) preference relation $\geqslant$ of a decision maker is a binary relation on $L$. As usual we write $x \leqslant y$ if $y \geqslant x, x>y$ if $x \geqslant y$ and not $y \geqslant x, x<y$ if $y>x$, and $x=y$ if both $x \geqslant y$ and $y \geqslant x$. In Section 3 we shall furthermore introduce a hypothetical preference relation $\geqslant$ on $L$, from which $\{,<, \equiv$ are derived analogously. We say $\geqslant$ is a weak order il it is transitive and complete (i.e. $x \geqslant y$ or $y \geq x$ for all $x, y)$. Then it also is reflexive, and $=$ is an equivalence relation.

Notation 2.1. For $x \in L, y, \in \mathcal{Y}_{i}, x_{-1} y_{j}$ is $\left(x\right.$ with $x$, replaced by $\left.y_{1}\right)$.

Definition 2.2. Coordinate $;$ is essential w.r.t. $\geqslant$ if there exist $x \in L, U, \in Y_{j}$ s.t. $x>x, 0$. Otherwise it is inessential.

KSV dealt with the following assumption, where a simple lottery on a set $X$ is a probability measure on $\left(X, 2^{\prime}\right)$, assigning probability 1 to a finite subset of $X$ (they in lact took $X$ finite):

Assumption 2.3. (Lottery Assumption). For every $j$, the set $\mathscr{C}_{j}$ is the set of simple lotteries on a non-empty set $X_{j}$ of 'prizes'.

Obviously $L$ is endowed with a 'mixture operation', assigning, to every $\alpha \in[0,1]$, and $x=\left(x_{1}, \ldots, x_{n}\right)$ and $y=\left(y_{1}, \ldots, y_{n}\right)$ in $L$, the simple lottery $(\alpha x+(1-\alpha) y=$ $\left(\alpha x_{1}+(1-\alpha) y_{1}, \ldots, \alpha x_{n}+(1-\alpha) y_{n}\right)$. Under the Lottery Assumption, we call $\geqslant$ continuous if, for every $x, y, z$ in $L$ with $x>y>z$. there exist $\alpha$ and $\beta$ in $(0,1)$ such 
that $\alpha z+(1-\alpha) x>y>\beta x+(1-\beta) z$; we call $\geqslant$ independent if, for every $x, y, z$ in $L$ and $\alpha$ in $(0,1), x>y$ implies $\alpha x+(1-\alpha) z>\alpha y+(1-\alpha) z$. A function $V,: \mathscr{\ell}_{j} \rightarrow \mathbb{R}$ is affine if $V_{j}\left(\alpha x_{j}+(1-\alpha) y_{j}\right)=\alpha V_{j}\left(x_{j}\right)+(1-\alpha) V_{j}\left(y_{j}\right)$, for all $x_{j}, y$, in $\gamma_{j}$, and all $\alpha \in[0,1]$.

Theorem 2.4. Under the Lotiery Assumption 2.3, the following two statements are equivalent:

Statement 2.4.i: 'There exist affine $V_{j}: \mathscr{C}_{j} \rightarrow \mathbb{R}, j=1, \ldots, n$, s.t.:

$$
\left|x \geqslant y \Leftrightarrow \sum_{j=1}^{n} V_{j}\left(x_{j}\right) \geq \sum_{j=1}^{n} V_{j}\left(y_{j}\right)\right| \text { for all } x, y \in L .
$$

Statement 2.4.ii: " $\geqslant$ is a continuous independent weak order'.

Furthermore, if Statement 2.4.i holds, then $\left(V_{j}\right)_{j=1}^{n}$ can be replaced by $\left(V_{j}^{\prime}\right)_{j=1}^{n}$ if and only if there exist real $\left(\beta_{i}\right)_{j=1}^{n}$, and positive $\alpha$, s.t. $V_{j}^{\prime}=\beta_{j}+\alpha V_{j}$ for all $j$.

We shall adapt the work of KSV to the case where, instead of the Lottery Assumption, we have:

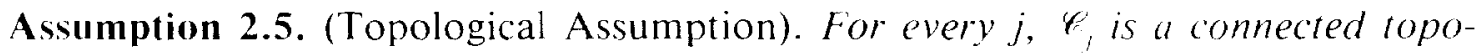
logical space. $L$ is endowed with the product topology.

In Kelley (1955) the reader can find definitions and basic results of topology. A reader, not interested in general topology, may simply assume that every $\mathscr{E}$, is a convex subset of a linear space, e.g. $\mathscr{\ell}_{,}=\mathbb{R}_{+}^{\prime \prime \prime}$, and consequences are commodity bundels, or $\mathscr{\ell}=\mathbb{R}$, and consequences are amounts of money. Under the Topological Assumption the preference relation $\geqslant$ is continous if $\{x \in L: x \geqslant y\}$ and $\{x \in L: x \leqslant y\}$ are closed, for all $y \in L$.

The following property is a central tool under the Topological Assumption.

Definition 2.6. $\geqslant$ is coordinate independent $(\mathrm{Cl})$ if

$$
x_{j} v_{j} \geqslant y_{-j} v_{j} \Leftrightarrow x_{-j} w_{j} \geqslant y_{-,} w_{j} \text { for all } j, x, y, v_{j}, w_{j} .
$$

CI means that the preference between $x$ and $y$ is independent of those coordinates where $x$ and $y$ are equal. It is known under various names as '(strong) separability', (part of the) 'sure-thing principle', '(preferential) independence'. Note that CI is implied by Statement 2.4.i, thus by Statement 2.4.ii as well. Under $\mathrm{Cl}$, the following definition of preference relations $\geqslant_{j}$ on $\mathscr{C}_{j}$ is of use:

Definition 2.7. For every $j, v_{j}$ and $w_{j} \in \mathscr{C}_{j}$ we write $v_{j} \geqslant, w_{j}$ if there exists $x \in L$ s.t. $x_{-j} v_{j} \geqslant x_{-j} w_{j}$.

Under CI we have $v_{j} \geqslant_{j} w_{j} \Leftrightarrow\left[x_{-j} v_{j} \geqslant x_{-j} w_{j}\right.$ for all $\left.x \in L\right]$, and every $\geqslant_{j}$ is a weak 
order if $\geqslant$ is; furthermore continous if $\geqslant$ is. The following result, a slight strengthening of Theorem 3 in Debreu (1960), and of Theorem 14 in Section 6.11.1 of Krantz et al. (1971), is proved in Wakker (1986, Theorem 4.1).

Theorem 2.8. Let the Topological Assumption 2.5 hold. Let at least three coordinates be essential w.r.t. ?. Then the following two statements are equivalent:

Statement 2.8.i: "There exist continuous $l_{j}: t_{j} \rightarrow, j=1, \ldots, n$, s.t.

$$
\left|x \geqslant y \Leftrightarrow \sum_{j=1}^{n} V_{i}\left(x_{j}\right) \geq \sum_{j=1}^{n} V_{j}(y)\right| \text { for all } x, y \in L:
$$

Statement 2.8.ii: '> is a continous CI weak order'.

Hurthermore, if Statement 2.8.i applies, then $\left(V_{i}\right)_{j=1}^{\prime \prime}$ can be replaced by $\left(V_{j}^{\prime}\right)_{j}^{\prime \prime}$, if and only if there exist real $\left(\beta_{j}\right)_{j=1}^{\prime \prime}$, and positive $\alpha$, s.t. $V_{j}=\beta_{j}+\alpha$, for all $j$.

Theorems 2.4 and 2.8 can be interpreted as characterizations of state dependent expected utility. There can always be thought to exist 'subjective probabilities' $(p,)_{3-1}^{\prime \prime}$ and 'state dependent utility functions' $\left(C_{,}\right)_{j=1}^{\prime \prime}$, s.t. $V=p, l$, for all $j$. Note however that from merely the preference relation (all information of which is contained in $\left.\left(V_{j}\right)_{j-1}^{\prime \prime}\right)$ one can never uncover uniquely what the $p_{i}$ 's and $U_{j}$ 's are. For that, further information is needed.

\section{Auxiliary hypothetical probabilities}

An example of further information, as mentioned above, is provided by KSV. Let $\left(j_{j}\right)_{j}^{\prime \prime}$, strictly positive, summing to one, be a hypothetical, to the decision maker conceivable, probability distribution on $S$. We suppose that we know $\geqslant$, the preference relation that the decision maker would have on $L$ if his subjective probabilities would be equal to $\left(\hat{p}_{j}\right)_{j=1}^{\prime \prime}$. And we are interested in the case where there exist $\left(p_{j}\right)_{j=1}^{\prime \prime},\left(U_{j}\right)_{j-1}^{\prime \prime}$ s.t. both $x \geqslant y \Leftrightarrow \Sigma_{j=1}^{\prime \prime} p_{j} U_{,}\left(x_{j}\right) \geq \Sigma_{j=1}^{\prime \prime} p_{j} U_{j}\left(y_{j}\right)$, and $x \geqslant y=$ $\Sigma^{\prime \prime}=1, \hat{p}_{,},\left(x_{j}\right) \geq \Sigma_{j=1}^{\prime \prime} \hat{p}_{j} U_{i}\left(y_{j}\right)$, for all $x, y$. In such a case the decision maker could be called consistent. Under the hypothetical probabilities his 'tastes', quantified by $\left(C_{j}^{\prime}\right)_{i}^{\prime \prime}$, have remained unaffected. Before continuing, for the sake of casy reference, we write out the analogue of Theorem 2.8 for $>$ instead of $>$.

Theorem 3.1. Let the Topological Assumption 2.5 hold. Let at least three coordinates be essential w.r.t. $\geqslant$. Then the following two statements are equivalent:

Statement 3.1.i: 'There exist continuous $\hat{V}_{1}: Y_{i} \rightarrow \mathbb{R}, j=1, \ldots, n$, s.l.

$$
\left|x \geqslant y \Leftrightarrow \sum_{i=1}^{n} \hat{V}_{i}\left(x_{j}\right) \geq \sum_{i=1}^{n} \hat{V}_{i}\left(y_{j}\right)\right| \text { for all } x, y \in L:
$$

Statement 3.1.ii: ' $\$$ is a continuous CI weak order'.

In KSV the following condition ('Consistency axiom'; reformulated for our con- 
text) was necessary and sufficient for consistency. Note for it that by positivity of the $\hat{p}_{j}$ 's, essentiality of $j$ w.r.t. $\geqslant$ under consistency implies essentiality of $j$ w.r.t.

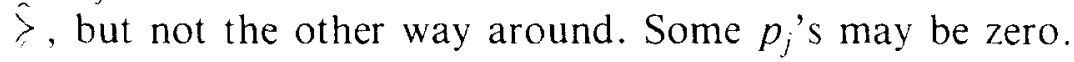

Definition 3.2. $\geqslant$ is ordinally consistent with $\geqslant$ if the following 1 wo conditions hold:

Condition 3.2. $a$ : For every $j, \geqslant j \supset \sum_{j}$.

Condition 3.2.b: For every j, essential w.r.t. $\geqslant, \sum_{j} \supset \geqslant$.

Obviously ordinal consistency holds if and only if $\geqslant_{1}=\sum_{\text {; }}$ for all $j$ essential w.r.t. ?. The following theorem gives the major part of the 'Main Theorem' of KSV; we omit their considerations concerning 'null states'.

Theorem 3.3. Let the Lottery Assumption 2.3 hold. Let $>$ be non-empty. Let $>$ and $\geqslant$ be continous independent weak orders. Let $\hat{p}_{1}, \ldots, \hat{p}_{n}$ be positive and sum to one. The following two statements are equivalent:

Statement 3.3.i: 'There exist affine $U_{j}: \mathscr{C}_{j} \rightarrow \mathbb{R}$ and $p_{j} \geq 0, j=1, \ldots, n$, with $\square p_{j}=1$, s.t. $x \geqslant y \Leftrightarrow \sum p_{j} U_{j}\left(x_{j}\right) \geq \sum p_{j} U_{j}\left(y_{j}\right)$ and $x \geqslant y \Leftrightarrow \sum \hat{p}_{j} U_{j}\left(x_{j}\right) \geq \sum \hat{p}_{j} U_{j}\left(y_{j}\right)$ for all $x, y$ in $L^{\prime}$.

Statement 3.3.ii: ' $\geqslant$ is ordinally consistent with $\geqslant$ '.

Furthermore, the ratio of $p_{k}$ and $p_{l}$ in Statement 3.3.i is uniquely determined for all $k$, l, essential w.r.t. $\geqslant$.

Obviously Statement 3.3.i implies Statement 2.4.i, with $V_{j}:=p$, $l$ for all $j$. The following Proposition shows that, under the Topological Assumption, ordinal consistency is too weak a requirement to imply the existence of the probabilities $p_{1}, \ldots, p_{n}$ as in Statement 3.3.i.

Proposition 3.4. Let the Topological Assumption 2.5 hold. Let Statements 2.8.i and 3.1.i hold. Then are equivalent:

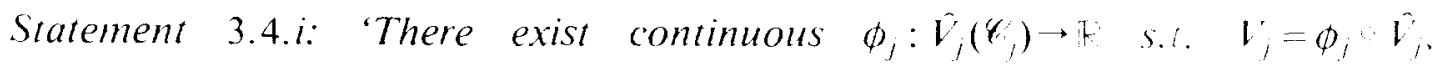
$j=1, \ldots, n$, where every $\phi_{j}$ is strictly increasing or constant.

Statement 3.4.ii: ' $\geqslant$ is ordinally consistent with $\geqslant$ '.

Proof. If $j$ is inessential w.r.t. $\geqslant$, then $l$, is constant, and evervthing follows. Next let $j$ be essential w.r.t. $\geqslant$. $V_{j}$ represents $\geqslant j$, so is not constant. Thus under (i) $\phi_{j}$ is strictly increasing, and $\geqslant_{j}=\geqslant$, follows. Under (ii), $V$, and $r$, represent the same $\geqslant_{j}=\xi_{j}$, hence $V_{j}=\phi_{j} \hat{V}_{j}$ for some strictly increasing $\phi_{j}$. Since $V_{j}\left(\psi_{j}\right)$ and $V_{,}\left(\psi_{j}\right)$ are connected, and the strictly increasing $\phi$, is onto $V_{\gamma}\left(\psi_{j}\right), \phi$, cannot make 'jumps'. It must be continuous.

Under the Lottery Assumption and the conditions of the 'KSV Theorem' 2.4, one deals with 'vNM-utility functions', i.e. the $V_{j}$ 's and $V_{j}$ 's are affine. Then the $\phi_{j}$ 's 
as above also will be affine, and the probabilities can be derived. In Statement 3.4.i, the $\phi_{j}$ 's may very well be non-affine, e.g. $\phi_{j}: \mu$-exp $(\mu)$. Hence a stronger condition than ordinal consistency seems needed for the topological context. We shall first show that in the KSV-result a slight weakening of ordinal consistency would already have sufficed to obtain the desired result. The cardinal consistency condition', to be introduced in the next section, will be a strengthening of this weakening.

I emma 3.5. In Statement 3.3.ii, ordinal consistency may be replaced by the Condition 3.2.a.

Proof. We show that, under the assumptions of Theorem 3.3. Condition 3.2.a implies Condition 3.2.b. So let for every $i, \geqslant_{i} \supset \geqslant_{i}$. And let $j$ be essential w.r.t. $\geqslant$, say $u_{j}>_{j} w_{j}$. Let further, for some $x_{j}, y_{j}, x_{j} \geqslant_{j} y_{j}$. To show is that $x_{j} \sum_{j} y_{j}$.

For any $a \in L$, we have $a_{-j} v_{j}>a_{-j} w_{j}$ and $a_{-j} x_{j} \geqslant a_{-j} y_{j}$, hence $a_{-j}\left(\alpha v_{j}+(1-\alpha) x_{j}\right)>$ $a_{-j}\left(\alpha w_{j}+(1-\alpha) y_{j}\right)$ for every $\alpha \in(0,1]$. So $\alpha v_{j}+(1-\alpha) x_{j}>_{j} \alpha w_{j}+(1-\alpha) y_{j}$. By Condition 3.2.a. $\left.\alpha v_{j}+(1-\alpha) x_{j}\right\rangle_{j} \alpha w_{j}+(1-\alpha) y_{j}$, for every $\alpha \in(0,1]$. Thus $a_{-j}\left(\alpha v_{j}+(1-\alpha) x_{j}\right)>a_{-j}\left(\alpha w_{j}+(1-\alpha) y_{j}\right)$. From continuity of $\bar{y}$ it can be derived that $a, x_{j} \geqslant a_{-j}, y_{j}$. It follows that $x, y_{j} y_{j}$.

The idea in the above Lemma, to derive (3.2.b) from one-sided monotonicity as (3.2.a), has been inferred from the Proof of Theorem 1 in Peters (1985).

\section{Cardinal consistency}

The condition that in our set-up is the necessary and sufficient criterion for verification/falsification of consistency is the following:

Definition 4.1. $\geqslant$ is cardinally consistent (CC) with $\geqslant$ if $\left[x, a_{i} \leqslant, b_{1}, x, c_{i} \geqslant y_{1}, d_{i}\right.$, and $\left.v, a_{i} \geqslant w_{-i}, b_{i}\right]$ implies $\left[v, c_{i} \geqslant w, d_{i}\right]$ for all $i, x, \ldots, d_{i}$.

The idea is to conclude from the first two $\geqslant$ preferences that, intuitively speaking, the intensity of preference of $c_{i}$ over $d_{i}$ is at least as large as that of $a_{i}$ over $b_{i}$, for as far as $\geqslant$ is concerned. Were now $v_{-} c_{i}<w_{-,} d_{1}$, then a same reasoning would give an opposite result for $\geqslant$. And that is forbidden by $\mathrm{CC}$. The idea, leading to $C C$, can be recognized in the "triple cancellation' condition in Krantz et al. (1971), the 'corresponding tradeoffs condition' in Keeney and Raiffa (1976), the 'Reidemeister condition' in Blaschke and Bol (1938), and in 'cardinal coordinate independence' of Wakker (1984).

Lemma 4.3.i will show that indeed cardinal consistency strengthens Condition 3.2. a of ordinal consistency. First we show that, under the Lottery Assumption, cardinal consistency is implied by ordinal consistency, in the context of the KSVTheorem 2.4. 
Proposition 4.2. Let the Lotterv Assumption 2.3 hold, and let Statement 2.4. ii hold. Let Condition (3.2.a) of ordinal consistency hold. Then $\geqslant$ is $\mathrm{CC}_{\text {with }}$.

Proof. Say we had $x_{-i} a_{i} \leqslant y_{-i} b_{i}, x_{-i} c_{i} \geqslant y_{-i} d_{i}, v_{-i} a_{i} \geqslant w_{-i} b_{i}$, and $v_{i} c_{i}<w_{-i} d_{i}$. The first two preferences imply $\left(\frac{1}{2} x+\frac{1}{2} y\right)_{-i}\left(\frac{1}{2} a_{i}+\frac{1}{2} d_{i}\right) \leqslant\left(\frac{1}{2} x+\frac{1}{2} y\right)_{-i}\left(\frac{1}{2} b+\frac{1}{2} c_{i}\right)$, i.e. $\frac{1}{2} a_{i}+\frac{1}{2} d_{i} \leqslant \frac{1}{2} b_{i}+\frac{1}{2} c_{i}$. The third and fourth preferences in this proof imply

$$
\left(\frac{1}{2} v+\frac{1}{2} w\right)_{-i}\left(\frac{1}{2} a_{i}+\frac{1}{2} d_{i}\right)>\left(\frac{1}{2} v+\frac{1}{2} w\right)_{-i}\left(\frac{1}{2} b_{i}+\frac{1}{2} c_{i}\right),
$$

i.e. $a_{i}+\frac{1}{2} d_{i}>_{i} \frac{1}{2} b_{i}+\frac{1}{2} c_{i}$. This and the $\xi_{i}$-preference above give violation of Condition 3.2.a in ordinal consistency.

Lemma 4.3. Let $\geqslant$ and $\geqslant$ be weak orders. let $\geqslant$ be CC with $\geqslant$. Then follows:

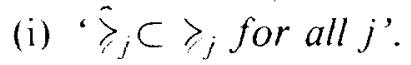

(ii) 'If $j$ is essential w.r.t. $\geqslant$, it is w.r.t. $\hat{?}$ '.

(iii) 'If the Topological Assumption 2.5, and Statements 2.8.i and 3.1.i hold, then continuous non-decreasing $\phi_{j}: \hat{V}_{j}\left(\mathscr{C}_{j}\right) \rightarrow \mathbb{R}$ exist s.t. $V_{j}=\phi_{i}: \hat{V}_{j}, j=1, \ldots, n$ '.

Proof. Let $x_{-j} v_{j} \geqslant x_{-j} w_{j}$. By $x_{-j} v_{j} \leqslant x_{-j} v_{j}, x, x_{j} \geqslant x, w, x, w_{,}, x, w_{1}$ and $\mathrm{C} C \mathrm{we}$ get $x, v, \geqslant x, w$. This implies (i) and (ii). For (iii), first suppose $f(x)=f,(y$,$) .$ Then $x_{j} \fallingdotseq y^{\prime}$, by twofold application of (i) we get $x_{j}=y_{1}$. Thus $l(x)=\hat{l}(y$,$) im$ plies $V_{j}\left(x_{j}\right)=V_{j}\left(y_{j}\right)$. There must exist $\phi_{j}$ s.t. $V_{j}=\phi_{,} V_{i}$. Nondecreasingness of $\phi$ is by (i), continuity is as in Proposition 3.4.

We shall need the following result from elementary analysis.

Lemma 4.4. Let $A \subset \mathbb{R}$ be convex. Let $\phi: A \rightarrow \mathbb{R}$ be continuous. Let $:>0$ and $\zeta>0$ exist s.t., for all $\alpha, \beta, \gamma, \delta$ in $A$ with $|\alpha-\beta| \leq \varepsilon$ and $\phi(\alpha)-\phi(\beta) \leq,[\alpha-\beta=\gamma-\delta]$ implies $\left[\phi(\alpha)-\phi(\beta)=\phi\left(\gamma^{\prime}\right)-\phi(\delta)\right]$. Then $\phi$ is affine.

Proof. We show $\phi$ has a second derivative 0 everywhere. Let $\mu \in A$. L ef $\mu>0$ be so small that $v \leq \varepsilon / 2$, and for all $\sigma, \mu$ in $A,|\sigma-\mu| \leq v \Rightarrow|\phi(\sigma)-\phi(\mu)| \leq-2$. Thus, with $\beta=\gamma=\alpha / 2+\delta / 2$ above, we get $\phi(\alpha / 2+\delta / 2)=\phi(\alpha) / 2+\phi(\delta) / 2$ for all $\alpha, \delta$ in $4 \cap[\mu-v, \mu+v]$. The continuous $\phi$ must be affine on $A \cap[u-v, \mu+1]$. So second derivative exists in $\mu$, and equals 0 .

The following proposition captures the meaning of $\mathrm{CC}$, without relating it yet to the hypothetical probabilities $\left(\hat{p}_{j}\right)_{i=1}^{\prime \prime}$.

Proposition 4.5. Let the Topological Assumption 2.5 hold. Let Statements 2.8.i and $3.1 . i$ hold. Let two or more coordinates be essential w.r.t. >. Then are equivalent:

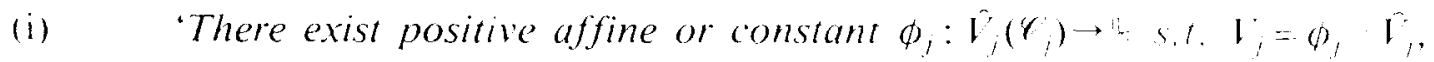
for all $j$.

(ii) ' $\geqslant$ is cardinally consistent with $\geqslant$ ? 


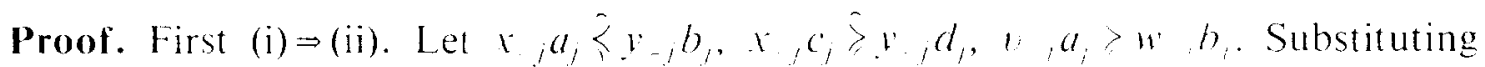
F.

$$
\hat{V}_{j}\left(a_{j}\right)-\hat{V}_{j}\left(b_{j}\right) \leq \sum_{i \neq j}\left[\hat{V}_{i}\left(y_{i}\right)-\hat{V}_{i}\left(x_{i}\right)\right] \leq \hat{V}_{j}\left(c_{j}\right)-\hat{V}_{j}\left(d_{j}\right)
$$

Thus

$$
V_{j}\left(c_{i}\right)-V_{j}\left(d_{i}\right) \geq V_{j}\left(a_{j}\right)-V_{j}\left(b_{i}\right) \geq \sum_{1 \neq 1}\left[V_{i}\left(w_{i}\right)-V_{i}\left(u_{i}\right)\right]
$$

the first inequality by constantness or positive affinity of $\phi$, the second since 1. $a_{j} \geqslant w_{j} b_{j}$. Now $v_{-j}, c_{j} \geqslant w_{-j} d_{j}$ follows.

Next (ii) $\Rightarrow$ (i). Let $j \leq n$ be arbitrary. Since there is another coordinate than $j$ that is essential w.r.t. $\geqslant$, and thus by Lemma 4.3.ii w.r.t. $;$, there exist $x, y, v, w$ s.t.

$$
\sum_{i \neq 1}\left[\hat{V}_{i}\left(x_{i}\right)-\hat{V}_{i}\left(y_{i}\right)\right]=: \varepsilon>0, \quad \sum_{i \neq j}\left[V_{i}\left(u_{i}\right)-V_{i}\left(w_{i}\right)\right]=: \therefore>0 .
$$

By continuity of $x \mapsto \Sigma_{i \neq 1}, V_{1}\left(x_{i}\right)$ and of $x-\Sigma_{i \neq j}, V\left(x_{i}\right)$, and connectedness of $L$, for

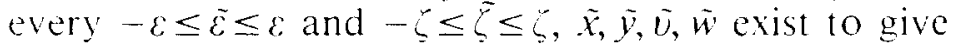

$$
\sum_{i \neq j}\left[\hat{V}_{i}\left(\tilde{x}_{i}\right)-\hat{V}_{i}\left(\tilde{l}_{i}\right)\right]=\bar{\varepsilon}, \quad \sum_{i \neq 1}\left[V_{i}\left(\tilde{u}_{i}\right)-V_{i}\left(\tilde{w}_{i}\right)\right]=
$$

Consider now the $\phi_{j}$ of Lemma 4.3.iii. Let in its convex domain $A:=\hat{\zeta}_{i}\left(\varphi_{i}\right)$ he given $\alpha, \beta, \gamma, \delta$ s.t. $-\varepsilon \leq \alpha-\beta=\gamma-\delta \leq \varepsilon$, and s.t. $-\zeta \leq \phi_{i}(\alpha)-\phi_{i}(\beta) \leq \zeta$. Let $a_{1}, b_{j}, c_{j}, d$, be s.t. $\hat{V}_{j}\left(a_{j}\right)=\alpha, \quad \hat{V}_{j}\left(b_{j}\right)=\beta, \quad \hat{V}_{j}\left(c_{j}\right)=\gamma, \quad \hat{V}_{j}\left(d_{j}\right)=\delta$. Take $\tilde{x}, \hat{y}$ s.t.

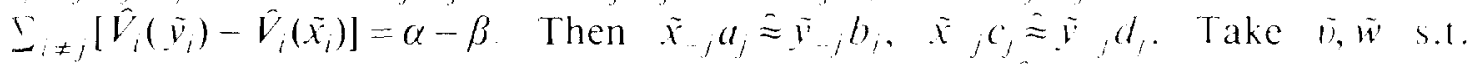
$\Sigma_{i \neq j}\left[V_{i}\left(\bar{u}_{i}\right)-V_{i}\left(\bar{w}_{l}\right)\right]=\phi_{j}(\beta)-\phi_{j}(\alpha)$. Then, since $V_{j}=\phi_{j} \dot{V}_{j}, \bar{l}_{j}, a_{i}=\bar{l}_{j}, b_{j}$. Twofold

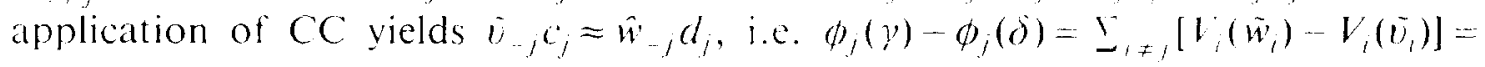
$\phi_{1}(\alpha)-\phi_{j}(\beta)$. Lemma 4.4 shows that $\phi_{j}$ is affine. From Lemma $4.3 . \mathrm{i}$ it can be derived that $\phi_{j}$ is non-decreasing, so either constant or positive atfine.

\section{Main result and conclusions}

Theorem 5.1. Let $n \in M,\left(\psi_{1}\right)_{j=1}^{\prime \prime}$ a sequence of connected topological spaces (e.g. $\left.Y_{i}=\mathbb{R}_{+}^{m_{j}}\right)$, $\geqslant$ and $\geqslant$ two binary relations on $L=X_{j=1}^{n} \mathscr{C}_{j}$, L endowed with the product lopology. Let $\left(\hat{p}_{j}\right)_{j-1}^{n}$ be a sequence of strictly positive real numbers, summing to one. Let at least three coordinates be essential w.r.t. $\gg$. Equiralent are:

'There exist nonnegative $\left(p_{j}\right)_{1}^{n}$, , summing to one. and continuous

$$
\begin{aligned}
& U_{j}: \mathscr{C}_{j} \rightarrow \mathbb{R}, j=1, \ldots, n, \text { s.t. } x \geqslant y \Leftrightarrow \sum_{j=1}^{n} p_{j} U_{j}\left(x_{j}\right) \geq \sum_{j=1}^{n} p_{j} U_{j}\left(y_{j}\right) \text { and } \\
& x \geqslant y \Leftrightarrow \sum_{j=1}^{n} \hat{p}_{j} U_{i}\left(x_{j}\right) \geq \sum_{j=1}^{n} \hat{p}_{j} U_{j}\left(y_{j}\right) \text { for all } x, y \in L:
\end{aligned}
$$

(ii) '”and $\geqslant$ are continuous coordinate independent weak orders, ? is cardinally consistent with $\geqslant$. 
Proof. That (i) implies (ii) is by the definitions $V_{j}:=p_{j} U_{j}, \hat{V}_{i}:=p_{j} U_{j}, \phi_{j}(\alpha):=$ $p_{j} \alpha / \hat{p}_{j}$, Proposition 4.5 and Theorems 2.8 and 3.1 .

Next we assume (ii), and derive (i). By Lemma 4.3.ii $\geqslant$ has at least three essential coordinates. Theorems 2.8 and 3.1 have statements (ii) satisfied, thus also statements (i). Now (i) in Proposition 4.5 follows. Hereby, for arbitrary fixed $z \in L$, there exist nonnegative $\left(\lambda_{j}\right)_{j=1}^{\prime \prime}$ s.t. for all $x$ and $j$ we have $V_{j}\left(x_{j}\right)-V_{j}\left(z_{j}\right)=\lambda_{j}\left[\vec{V}\left(x_{j}\right)-\vec{V}_{j}^{\prime}\left(z_{j}\right)\right]$. At least three coordinates are essential w.r.t. $\geqslant$, so at least one (even three) $\lambda_{j}$ 's are nonzero. Consequently $0<v:=\Sigma_{j-1}^{\prime \prime} \hat{p}_{j} \lambda_{j}$. We define $U_{j}:=\left[\hat{V}_{1}-\hat{V}_{j}\left(z_{j}\right)\right] / \hat{p}_{j}$, and $p_{j}=\lambda_{j} \hat{p}_{j} / v$ for all $j$. Thus $\hat{V}_{j}=\hat{p}_{j} U_{j}+\hat{V}_{j}\left(z_{j}\right), V_{j}=v p_{j} U_{j}+V_{j}\left(z_{j}\right)$, for all $j$.

In (i) above $\left(U_{j}\right)_{j=1}^{n}$ can be replaced by $\left(\bar{U}_{j}\right)_{j=1}^{n}$ iff real $\left(\beta_{j}\right)_{j-1}^{\prime \prime}$, and positive $\alpha$, exist s.t. $\bar{U}_{j}=\beta_{j}+\alpha U_{j}$ for all $j$. The uniqueness result w.r.t. $\left(p_{j}\right)_{j=1}^{\prime \prime}$ is not so simple. On the set of essential coordinates w.r.t. $\geqslant$, the ratios of the $p_{j}$ 's are uniquely determined. For the coordinates, inessential w.r.t $\overrightarrow{\geqslant}$, so those with constant $\hat{v_{i}}$, and $U_{j}$, the $p_{j}$ 's are arbitrary. As long as the $p_{j}$ 's are nonnegative, and sum to one, of course. This can be seen in the above proof: after $\left(V_{j}\right)_{j=1}^{\prime \prime}$ and $\left(\hat{V}_{j}\right)_{j}^{\prime \prime}$, are chosen, for the constant $\hat{V}_{j}$ 's the $\lambda_{j}$ 's can be arbitrarily chosen, for the nonconstant $\hat{V}_{j}$ 's they are uniquely determined.

For interpretations of results as above the reader is referred to $\mathrm{KSV}$. They also indicate interesting implications for statistics.

Schmeidler noted that in (ii) above $\mathrm{CI}$ for $\geqslant$ may be derived from $\mathrm{CC}$. This is by the choice $x=y, a_{i}=b_{i}, c_{i}=d_{i}$ in Definition 4.1 . Also we could have weakened $\mathrm{CC}$ by requiring it only for $b_{i}=c_{i}$. Note that Lemma 4.3 did not yet give ordinal consistency. This now follows from (i) above. For the case of two essential coordinates in (ii) above we would have had to add in (ii) above for $\geqslant$ and $\geqslant$ the 'Thomsen Condition'. For the definition of this the reader is referred to Krantz et al. (1971). The case of one essential coordinate for $\geqslant$ is somewhat different. This refers in fact to the case where there is no uncertainty, hence is uninteresting for our purposes.

Let us finally remark that, for recovering from $\geqslant$, via $\left(V_{j}\right)_{j=1}^{n}$, and $\left(\hat{p}_{j}\right)_{j=1}^{n}$, the probabilities $\left(p_{j}\right)_{j=1}^{n}$, it is not necessary to know all of $\geqslant$. For instance if (not $\left.b_{2} \hat{\approx}_{2} a_{2}\right)$ and $\left(x_{-1} a_{1}\right)_{-2} a_{2} \approx\left(x_{-1} b_{1}\right)_{-2} b_{2}$, then $\hat{p}_{1}\left[U_{1}\left(a_{1}\right)-U_{1}\left(b_{1}\right)\right]=\hat{p}_{2}\left[U_{2}\left(b_{2}\right)-U_{2}\left(a_{2}\right)\right]$ so $\left[U_{1}\left(a_{1}\right)-U_{1}\left(b_{1}\right)\right] /\left[U_{2}\left(b_{2}\right) \cdots U_{2}\left(a_{2}\right)\right]=\hat{p}_{2} / \hat{p}_{1}$, and now from $\hat{p}_{1} p_{2}\left[V_{1}\left(a_{1}\right)-V_{1}\left(b_{1}\right)\right]=$ $p_{1} \hat{p}_{2}\left[V_{2}\left(b_{2}\right)-V_{2}\left(a_{2}\right)\right]$ we can already uncover the ratio of $p_{1}$ and $p_{2}$, for nonconstant $V_{1}$ and $V_{2}$.

\section{Acknowledgement}

D. Schmeidler and E. Karni stimulated the author to study this subject. They, and an anonymous referee, made useful remarks. 


\section{References}

K.J. Arrow, Optimal insurance and generalized deductibles, Scandinavian Actuarial J. (1974) 1-42.

W. Blaschke and G. Boll, Geometrie der Gewebe (Springer-Verlag, Berlin, 1938).

P.J. Cook and D.A. Graham, The demand for insurance and protection: The case of irreplaceable commodities, Quart. J. Econom. 91 (1977) 143-156.

G. Debreu, Topological methods in cardinal utility theory, in: K.J. Arrow, S. Karlin and P. Suppes, eds., Mathematical Methods in the Social Sciences (Stanford University Press, Stanford, 1960) pp. 16-26.

K. Eisner and R.H. Strotz, Flight insurance and the theory of choice. J. Polit Econom. 69 (1961) $350-368$.

1.N. Herstein and J. Milnor, An axiomatic approach to measurable utility, Econometrica 21 (1953) $291-297$.

E. Karni, Decision-making under Uncertainty: The Case of State-Dependent Preferences (Harvard . University Press, Cambridge, Massachussets, 1985).

E. Karni. D. Schmeidler and K. Vind, On state dependent preferences and subjective probabilities, Econometrica 5i (1983) 1021-1031.

R.L. Keeney and H. Raiffa, Decisions with Multiple Objectives (Wiley, New York, 1976).

I.L. Kelley, General Topology (American Book Company, New York, 1955)

D.H. Krantz, R.D. Luce, P. Suppes and A. Tversky, Foundations of Measurement, Vol. I (Academic Press, New York, 1971).

H.J.M. Peters, A note on additive utility and bargaining, Econ. Letters 17 (1985) 219-222.

1.P. Ramsey, Truth and Probability, in: The Foundations of Mathematics and other Logical Essays (Hartcourt, Brace, New York, 1931).

V.E. Yaari, Some remarks on measures of risk aversion and on their uses. 1. Econ. Theory 1 (1969) $315-329$.

P.P. Wakker, Cardinal coordinate independence for expected utility, J. Math. Psvch. 28 (1984) 110-117.

P.P. Wakker, Continuity of additively decomposable functions, Report 8610 (1986) Department of Mathematics, University of Nijmegen, The Netherlands. 